\title{
In vitro evaluation of the activity of an essential oil from Pistacia vera L. variety Bronte hull against Candida sp.
}

\author{
Manuela D'Arrigo ${ }^{1}$, Carlo Bisignano ${ }^{2}$, Pierangela Irrera ${ }^{3}$, Antonella Smeriglio ${ }^{1}$, Roberto Zagami ${ }^{1}$ \\ Domenico Trombetta ${ }^{1}$, Orazio Romeo ${ }^{1,4}$ and Giuseppina Mandalari ${ }^{1 *}$ (i)
}

\begin{abstract}
Background: Candida sp. represent the most common cause of fungal infections worldwide. In the present work, we have evaluated the activity of an essential oil extracted from pistachio hulls against a number of standard and clinical strains of Candida sp.

Methods: C. albicans ATCC 64550, C. parapsilosis ATCC 22019, 4 clinical strains of C. albicans, 3 clinical strains of C. parapsilosis and 3 clinical strains of C. glabrata were used. All clinical isolates were identified by species-specific PCRbased methods. Susceptibility studies were performed using pistachio hull essential oil alone or in combination with antifungal compounds. The interactions between pistachio hull essential oil and selected antifungal compounds were also evaluated using the checkerboard method and the mechanisms of interaction investigated by droplet size distribution.

Results: Pistachio hull essential oil was fungicidal at the concentrations between 2.50 and $5.0 \mathrm{mg} / \mathrm{ml}$. D-limonene and 3-Carene were the components with major activity. An antagonistic effect was observed with all combinations tested.

Conclusion: The antifungal activity of pistachio hull essential oil could be used to help control resistance in Candida species. More studies need to be performed to elucidate the mechanisms responsible for the activity of pistachio hull essential oil.
\end{abstract}

Keywords: Pistachio, Essential oil, Candida sp., Antifungal

\section{Background}

Over the last few years, incidence of Candida spp. infections have significantly increased, with higher mortality rates and hospital acquired infections [1]. In immunocompetent subjects, Candida spp. are generally responsible for mucosal infections, including thrush and vaginitis, which can lead to invasive candidiasis in immunocompromised patients, with emerging species becoming multi-drug resistant [2]. Due to the increased resistance to antifungal compounds, more effort is focused on natural drugs, to use alone or in combination with existing antimicrobials [3]. Essential oils (EOs) are a complex mixture of hydrocarbons and oxygenated

\footnotetext{
* Correspondence: gmandalari@unime.it

${ }^{1}$ Department of Chemical, Biological, Pharmaceutical and Environmental Science, University of Messina, Via SS. Annunziata, 98168 Messina, Italy Full list of author information is available at the end of the article
}

hydrocarbons which have recently gained interest due to their antimicrobial potential against human pathogens and their protective role regarding cardiovascular and neurological diseases, diabetes and cancer [4].

Gucwa et al. [5] have recently reported the activity of EOs from Thymus vulgaris, Citrus limonum, Pelargonium graveolens, Cinnamomum cassia, Ocimum basilicum and Eugenia caryophyllus against 183 clinical isolates of $C$. albicans and 76 isolates of $C$. glabrata. The chemical composition and antifungal effects of the Eugenia uniflora EO were recently investigated against C. albicans, C. krusei and C. tropicalis standard strains [6].

We have previously characterised the nutraceutical, antioxidant and cytoprotective activity of pistachio (Pistacia vera L., variety Bronte) hulls extracts: they are rich in phenols, including flavonols, phenolic acid, and flavan-3-ols, and anthocyanins, such as cyanidyn-3-O-galactoside $[7,8]$.

(c) The Author(s). 2019 Open Access This article is distributed under the terms of the Creative Commons Attribution 4.0 International License (http://creativecommons.org/licenses/by/4.0/), which permits unrestricted use, distribution, and reproduction in any medium, provided you give appropriate credit to the original author(s) and the source, provide a link to the Creative Commons license, and indicate if changes were made. The Creative Commons Public Domain Dedication waiver (http://creativecommons.org/publicdomain/zero/1.0/) applies to the data made available in this article, unless otherwise stated. 
Furthermore, the antimicrobial activity of the EO from pistachio hulls against Gram-positive and Gram-negative strains, both American Type Culture Collection (ATCC) and clinical isolates, was investigated [9].

In the present study, the EO extracted from pistachio hulls was tested against standard and clinical strains of C. albicans, C. glabrata and C. parapsilopsis, both alone and in combination with antifungal drugs. The molecular identification of the clinical strains is also reported.

\section{Methods}

\section{Plant material}

The hulls of ripe pistachio nuts (Pistacia vera L., Bronte variety) were collected in end-August 2017 by a local farmer in Bronte (Catania, Italy) and identified by Prof. Laura Cornara (Botanist at the Dept. DISTAV of the University of Genoa, Italy). A voucher specimen has been deposited in the ChiBioFarAm Department, University of Messina (Messina, Italy).

\section{Essential oil isolation and characterization}

The pistachio hull essential oil was isolated by hydrodistillation using a Clevenger apparatus and characterized by GC-FID and GC-MS analysis according to Smeriglio et al. [9].

\section{Origin and identification of the Candida strains}

All clinical Candida isolates tested in this study were recovered at the IRCCS Centro Neurolesi "Bonino-Pulejo" hospital, Messina, Italy, during a surveillance program funded by the Italian Ministry of Health for the prevention and control of healthcare-associated fungal infections (project code: GR-2011-02347606). These isolates were obtained from blood samples of patients with acquired brain injuries. Initially, all strains were presumptively identified using Candida medium (Becton Dickinson, Italy) and Vitek 2 yeast identification system (bioMérieux, Italy) following manufacturer's recommendations. The identity of the yeast isolates was subsequently confirmed by using simple and rapid species-specific PCR-based methods according to previous studies [10-12]. Briefly, total genomic DNA was extracted from Candida cells using the glass-beads disruption method followed by conventional phenol/ chloroform/isoamyl alcohol purification [13]. In vitro amplifications (total volume $50 \mu \mathrm{l}$ ) were carried out separately for each strain using the Dream Taq Green PCR Master Mix (Thermo Fischer scientific, Milan, Italy), a ready-to-use solution containing all reagents required for PCR to which were only added the genomic DNA template $(0.5 \mu \mathrm{g})$ and the specific primers $(0.5 \mu \mathrm{M}$ each), depending on the assay type (Table 1 ). The amplicons were analyzed by $1.5 \%$ agarose gel electrophoresis for determining the expected DNA fragment sizes for $C$. albicans, C. parapsilosis and C. glabrata (Table 1) by excluding the probable cryptic presence of phylogenetically closely related species.

\section{Microbial strains and culture conditions}

The following strains were used for the antifungal testing: C. albicans ATCC 64550, C. parapsilosis ATCC 22019, 4 clinical strains of $C$. albicans $(12,13,16,17), 3$ clinical strains of $C$. parapsilosis (26, 30, 34), 3 clinical strains of $C$. glabrata $(9,25,33)$. Strains were grown in RPMI 1640 (Sigma, Italy) at $30^{\circ} \mathrm{C}$ for $24 \mathrm{~h}$. For minimal fungicidal determination and killing curves, Sabouraud Dextrose Agar (Oxoid) was used.

\section{Susceptibility studies}

For the susceptibility studies, the EO from pistachio hull was dissolved in DMSO at the concentration of $10 \mathrm{mg} /$ $\mathrm{ml}$. The minimum inhibitory concentration (MIC) and the minimum fungicidal concentration (MFC) of pistachio hull EO and the antifungal compounds voriconazole, fluconazole and caspofungin (Sigma Aldrich, Italy) against the strains reported above were determined following the CLSI guidelines (M27-A3 2008, [14]). Serial dilutions were performed in RPMI 1640 at concentrations between 16 and $0.0156 \mu \mathrm{g} \mathrm{ml}^{-1}$ (voriconazole), 64 and $0.0625 \mu \mathrm{g} \mathrm{ml}^{-1}$ (fluconazole), 2 and $0.00195 \mu \mathrm{g} \mathrm{ml}^{-1}$ (caspofungin) and 10 and $0.0049 \% \mathrm{mg} / \mathrm{ml}$ (pistachio hull essential oil). MFC (minimal fungicidal concentration) for the pistachio hull EO was determined transferring each clear sample $(20 \mu \mathrm{L})$ on agar plate incubated at $30^{\circ}$ $\mathrm{C}$ for $48 \mathrm{~h}$. The MFC was defined as the lowest extract concentration that killed $99.9 \%$ of the final inocula after 24-48 $\mathrm{h}$ incubation.

In the combination assays, the 'checkerboard' procedure was followed [15] in order to test the efficacy of the combination EO/antifungal compounds against all tested strains. This method allows varying the concentrations of each antimicrobial along the different axes, thus ensuring that each well contained a different combination [16]. MIC data for pistachio hull EO and each antifungal compound were converted into fractional inhibitory concentration (FIC), defined as the ratio of the concentration of the antimicrobial in an inhibitory concentration with a second compound to the concentration of the antimicrobial by itself.

$\mathrm{FICI}=\mathrm{MIC}$ of A with $\mathrm{B} / \mathrm{MIC}$ of A.

In order to identify the active antifungal components of the pistachio hull EO, MICs and MFCs were also determined with $\alpha$-pinene, $\alpha$-terpineol, camphene, $\mathrm{D}$-limonene and 3-carene as well as the mix of these compounds at the concentrations found in the EO against C. albicans strain 16, C. glabrata strain 9 and $C$. parapsilosis strain 26 . 
Table 1 PCR primers used for molecular identification of Candida species tested in this study

\begin{tabular}{|c|c|c|c|c|c|}
\hline Molecular method & Species identified & Primer name & Sequence $\left(5^{\prime} \rightarrow 3^{\prime}\right)$ & Amplicon size & Reference \\
\hline \multirow[t]{2}{*}{ Singleplex PCR } & \multirow[t]{2}{*}{ C. albicans } & CR-f & GCTACCACTTCAGAATCATCATC & \multirow[t]{2}{*}{$\sim 960 \mathrm{bp}$} & \multirow[t]{2}{*}{ Romeo and Criseo, 2008} \\
\hline & & CR-r & GCACCTTCAGTCGTAGAGACG & & \\
\hline \multirow[t]{4}{*}{ Multiplex PCR } & \multirow[t]{4}{*}{ C. glabrata } & UNI-5,8S & ACCAGAGGGCGCAATGTG & \multirow[t]{4}{*}{$\sim 397 \mathrm{bp}$} & \multirow[t]{4}{*}{ Romeo et al., 2009} \\
\hline & & GLA-f & CGGTTGGTGGGTGTTCTGC & & \\
\hline & & NIV-f & AGGGAGGAGTTTGTATCTITCAAC & & \\
\hline & & BRA-f & GGGACGGTAAGTCTCCCG & & \\
\hline \multirow[t]{5}{*}{ Multiplex PCR } & \multirow[t]{5}{*}{ C. parapsilosis } & mCPF & TTGGCTTGGTAGGCCTTCTA & \multirow[t]{5}{*}{$\sim 171 \mathrm{bp}$} & \multirow[t]{5}{*}{ Asadzadeh et al., 2015} \\
\hline & & mCOF & TAAGTCAACTGATTAACTAAT & & \\
\hline & & mCMF & AACTGCAATCCTITCTITCTA & & \\
\hline & & mLEF & TACAGAATITTGAGAATTGTG & & \\
\hline & & $\mathrm{mCPCR}$ & AATATCTGCAATTCATATTACT & & \\
\hline
\end{tabular}

All experiments were performed in triplicate on three independent days.

\section{Time kill curves}

Tubes containing each antifungal compound at concentrations corresponding to $1,1 / 2$ and $1 / 4 \times$ MIC were inoculated with a suspension of C. glabrata strain 9, C. albicans strain 16 and C. parapsilosis strain 26, yielding a final fungal density of $5 \times 10^{5}$ $\mathrm{CFU} / \mathrm{ml}$ and then incubated at $30^{\circ} \mathrm{C}$ in a shaking incubator. A growth control was also performed. Samples for viable counting were withdrawn at $0,1,2,4$, 8 and $24 \mathrm{~h}$ and, if necessary, diluted in fresh medium. At least four dilutions of each sample were spread on agar plates (Sabouraud Dextrose Agar), incubated at $30^{\circ} \mathrm{C}$ and counted after $48 \mathrm{~h}$.

\section{Droplet size and size distribution}

Size distribution and average diameter of emulsions' droplets were determined by dynamic light scattering on a Malvern 4700 submicron analyzer (Malvern Instruments Inc., Worcestershire, U.K.). Distilled water was used as dispersant to avoid effects of multiple scattering, dispersion, and interactions between droplets. The cumulative mean diameter (z-average) and polydispersity index (PdI) were used to describe droplet average size and size distribution, respectively.

\section{Results}

\section{Antifungal activity of pistachio hull EO}

MICs and MFCs values for pistachio hull EO and three antifungal compounds were determined (Table 2). Results of negative controls using DMSO as a solvent at the concentration of $1 \%(v / v)$ indicated the complete

Table 2 MICs and MFCs of Pistachio Essential Oil (expressed as mg/ml) and antifungal compounds (expressed as $\mu \mathrm{g} / \mathrm{ml}$ ) against Candida sp.

\begin{tabular}{|c|c|c|c|c|c|c|c|c|}
\hline \multirow[t]{2}{*}{ STRAIN } & \multicolumn{2}{|c|}{ VORICONAZOLE } & \multicolumn{2}{|c|}{ FLUCONAZOLE } & \multicolumn{2}{|l|}{ CASPOFUNGIN } & \multicolumn{2}{|c|}{ Pistachio Essential Oil } \\
\hline & MIC & MFC & $\mathrm{MIC}$ & MFC & $\mathrm{MIC}$ & MFC & $\mathrm{MIC}$ & MFC \\
\hline Candida glabrata strain 9 & $0.015-0.031$ & 1 & $8-8$ & 8 & $0.015-0.031$ & 1 & $2.5-5.0$ & 5.0 \\
\hline Candida glabrata strain 25 & $0.015-0.031$ & 0.031 & $0.25-0.5$ & 0.5 & $0.125-0.25$ & 1 & 5.0 & 5.0 \\
\hline Candida glabrata strain 33 & $>16$ & / & $>64$ & / & $0.125-0.25$ & 1 & $1.25-2.50$ & 5.0 \\
\hline Candida parapsilosis strain 26 & $0.015-0.031$ & 0.031 & $0.5-1$ & 1 & $0.5-0.25$ & 0.5 & $1.25-2.50$ & 2.50 \\
\hline Candida parapsilosis strain 30 & $0.015-0.031$ & 0.031 & $0.5-1$ & 1 & $0.125-0.25$ & 0.5 & $1.25-2.50$ & 2.50 \\
\hline Candida parapsilosis strain 34 & $0.125-0.25$ & 0.25 & $8-4$ & 8 & $0.25-0.5$ & 0.5 & $1.25-2.50$ & 2.50 \\
\hline Candida albicans strain 12 & $>16$ & / & $>64$ & / & $0.0075-0.015$ & 0.015 & 5.0 & 5.0 \\
\hline Candida albicans strain 13 & $>16$ & / & $>64$ & / & $\leq 0.0019$ & / & 5.0 & 5.0 \\
\hline Candida albicans strain 16 & $>16$ & / & $>64$ & / & 0.015 & 0.015 & 5.0 & 5.0 \\
\hline Candida albicans strain 17 & $>16$ & / & $>64$ & / & $\leq 0.0019$ & / & 5.0 & 5.0 \\
\hline Candida albicans ATCC 64550 & 1 & 1 & 32 & 32 & 0.0625 & 0.0625 & $2.50-5.0$ & 5.0 \\
\hline Candida parapsilosis ATCC 22019 & 0.062 & 0.062 & 4 & 8 & $1-2$ & 1 & $1.25-2.50$ & 2.50 \\
\hline
\end{tabular}

MIC Minimum Inhibitory Concentration, MFC Minimum Fungicidal Concentration 
absence of inhibition of all the strains tested (data not shown). Pistachio hull EO was active against all strains tested, $C$. parapsilosis strains being the most sensitive (complete inhibition achieved with a concentration of $1.25-2.50 \mathrm{mg} / \mathrm{ml})$, followed by $C$. glabrata strains (complete inhibition achieved with a concentration of $1.25-5.0 \mathrm{mg} / \mathrm{ml}$ ) and C. albicans strains (complete inhibition achieved with a concentration of $5.0 \mathrm{mg} / \mathrm{ml})$. The effect of pistachio hull EO was fungicidal against all tested strains. Interestingly, all clinical strains of C. albicans and1 strain of C. glabrata (strain 33) were resistant to both voriconazole and fluconazole.

In the combination assays, the FIC index calculated for pistachio hull EO and each antifungal compound was $>4$ against all tested strains. Although the interpretation of the FIC indices depends on which definition is used, here we have interpreted the index as synergistic if the FIC index is $\leq 0.5$, additive or indifferent if $>0.5$ but $\leq 4$ and antagonistic if $>4[16,17]$.

Table 3 reports the MIC data for the pure compounds present in pistachio hull EO and the mix of the most representative compounds in the proportion found in the oil. Amongst the pure molecules, 3-carene was the most effective against the three representative tested strains, followed by D-limonene. No activity was detected with $\alpha$-pinene, $\alpha$-terpineol and camphene. Interestingly, the mix of compounds was effective against all tested strains, indicating a possible synergistic interaction amongst the individual compounds. As shown in Table 2, C. albicans strain 16 was the most resistant, followed by C. glabrata strain 9 and C. parapsilosis strain 26 .

\section{Fungal killing}

Concentration-dependent killing was observed with pistachio hull EO against all tested strains (Fig. 1 a-c). A good fungicidal effect was achieved after $2 \mathrm{~h}$ exposure against $C$. parapsilosis strain 26 at the concentration of $1 \mathrm{x}$ MIC, whereas the same effect was achieved against C. albicans strain 16 and C. glabrata strain 9 after $8 \mathrm{~h}$ exposure. Concentrations of $1 / 2$ MIC and $1 / 4$ MIC exerted a fungistatic effect against all tested strains. As reported in Tables 1 and 2, C. albicans strain 16 was the most resistant.

\section{Light scattering}

Table 4 summarizes the effect of the essential oil (EO) on the droplet size and emulsion polydispersity (PdI) in bacteria culture medium, taking into account both the hydrodynamic then the distribution width of this complex system. Both parameters are calculated according to the International Standard on dynamic light scattering, ISO 22412 [18]. The partition coefficient $(\log \mathrm{P})$ of the three antifungals under study was calculated by miLogP2.2 software [19].

The droplet size distribution of the different emulsions revealed the lack of a droplet population below $100 \mathrm{~nm}$, while a droplet population with a diameter above than $300 \mathrm{~nm}$ was detected.

Pistachio hull EO, fluconazole and voriconazole showed an average diameter greater than $1500 \mathrm{~nm}$ and a PdI equal to 1.0, whereas caspofungin showed an average diameter around $500 \mathrm{~nm}$ and a PdI of 0.679 .

\section{Discussion}

The present study has demonstrated that an essential oil extracted from pistachio hulls was effective against clinical strains of Candida. The dramatic rise in antimicrobial resistance is now considered a major health treat, with an estimated 700,000 people dying every year from antibiotic, antiviral, antifungal and antimalarial resistance infections [20]. Therefore, natural products have become an important source for drug development. Geraniol has been tested against $C$. albicans species and its mechanism of action has been elucidated [21]. Ebani et al. [22] have recently demonstrated the activity of five essential oils (star anise, basil, oregano, clary sage and thymus) against multidrug-resistant strains of Escherichia coli, Enterococcus spp., C. albicans and C. famata responsible for urinary tract infections. The pistachio hull essential oil tested in the present work was effective against Candida sp., whereas polyphenol-rich extracts from pistachios (natural raw shelled and roasted salted pistachios) and white grape juice used in our previous investigations were not effective against yeasts [23, 24]. Amongst the major components identified in the pistachio hull EO, D-limonene and 3-carene were effective against C. albicans strain 16, C. glabrata strain 9 and $C$. parapsilosis strain 26 (Table 3). This is in agreement

Table 3 MICs of pistachio hull EO pure compounds and their mix C. albicans strain 16, C. glabrata strain 9 and C. parapsilosis strain 26

\begin{tabular}{|c|c|c|c|c|c|c|}
\hline Strain & a-pinene & a-terpineol & Camphene & D-limonene & 3-Carene & Mix \\
\hline C. albicans strain 16 & $>1000$ & $>1000$ & $>1000$ & $125-250$ & $62.50-125$ & 250 \\
\hline C. glabrata strain 9 & $>1000$ & $>1000$ & $>1000$ & 62.50 & $62.50-125$ & $125-250$ \\
\hline C. parapsilosis strain 26 & $>1000$ & $>1000$ & $>1000$ & $31.25-62.50$ & $15.65-31.25$ & 62.5 \\
\hline
\end{tabular}

Values are expressed as $\mu \mathrm{g} / \mathrm{ml}$ and represent the mean of three determinations. Standard errors between these three determinations are negligible 

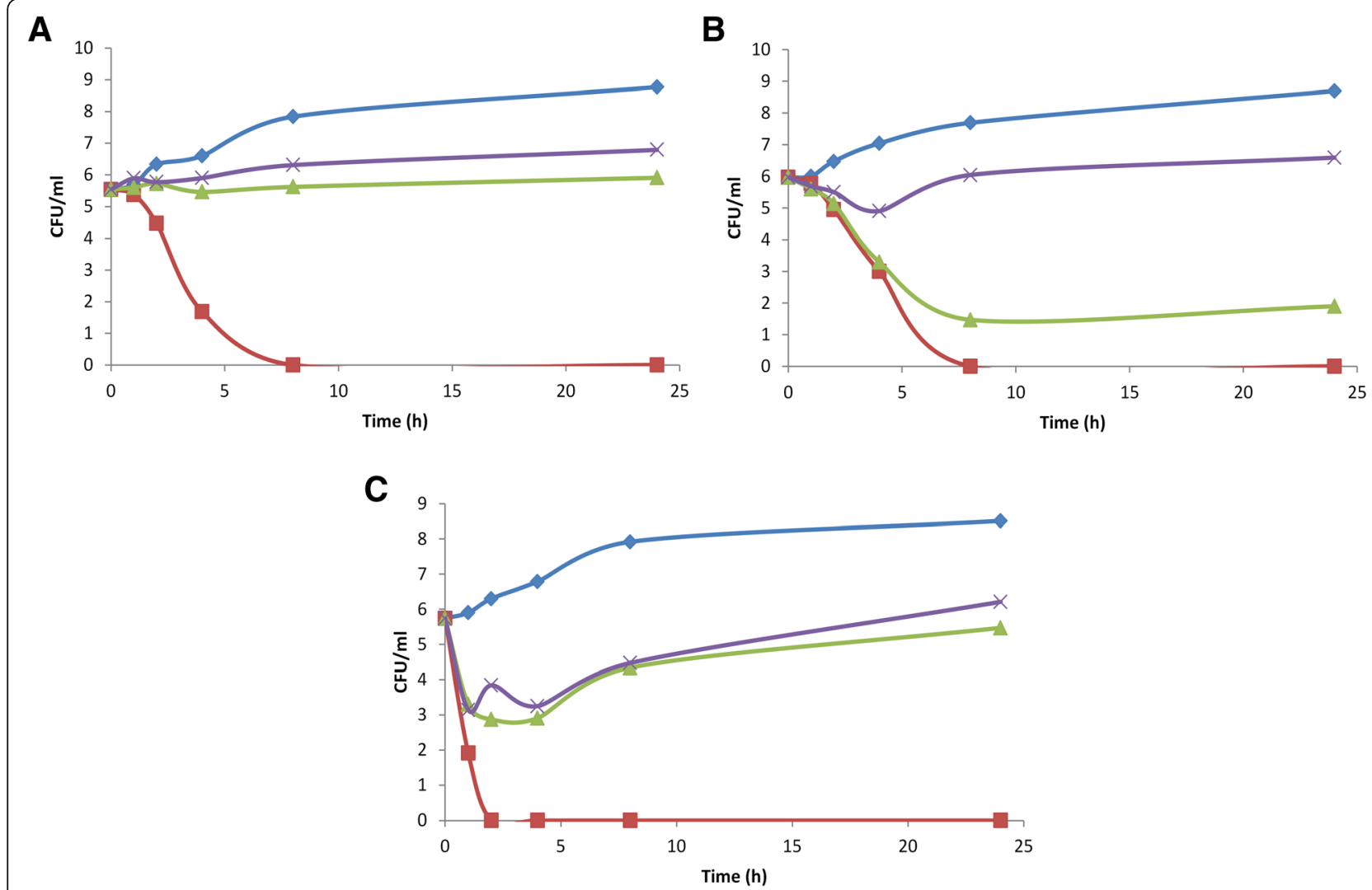

Fig. 1 Killing curves for pistachio hull EO against C. albicans strain 16 (a), C. glabrata strain 9 (b) and C. parapsilosis strain 26 (c). Control, $11 /$ $4 \times M I C, x ; 1 / 2 x M I C, \mathbf{\Delta} ; 1 \times M I C$, . Mean of three experiments. MIC = minimum inhibitory concentration

with the demonstrated antifungal activity against $C$. albicans of the Chamaecyparis nootkatensis essential oil [25].

It is known that plant natural products mostly exert their antifungal effects by membrane-active mechanism and synergistic effects can be found between different classes of plant products as well as between natural products and azoles [26]. Bioactive compounds present

Table 4 Effect of the Pistachio Essential oil on droplet size (ZAverage Diameter) and emulsion polydispersity (Polydispersity index) in yeast culture medium

\begin{tabular}{llll}
\hline Preparation & $\begin{array}{l}\text { Z-Average Diameter } \\
(\mathrm{nm})\end{array}$ & Polydispersity index & LogP \\
\hline RPMI+DMSO & $881.4 \pm 1.8$ & $0.836 \pm 0.011$ & - \\
Fluconazole & $1570.0 \pm 2.1$ & $1.0 \pm 0.012$ & -0.12 \\
Voriconazole & $2316.0 \pm 2.3$ & $1.0 \pm 0.011$ & 1.49 \\
Caspofungin & $531.3 \pm 1.6$ & $0.679 \pm 0.010$ & -4.59 \\
EO & $2151.0 \pm 2.2$ & $1.0 \pm 0.012$ & - \\
EO + Fluconazole & $847.5 \pm 2.3$ & $0.688 \pm 0.008$ & - \\
EO + Voriconazole & $343.7 \pm 1.5$ & $0.426 \pm 0.007$ & - \\
EO + Caspofungin & $690.1 \pm 2.1$ & $0.538 \pm 0.004$ & - \\
\hline
\end{tabular}

Theoretical $\log \mathrm{P}$ value of the three antifungals are reported. Each measurement represents mean \pm standard deviation $(n=3)$ in natural products, such as EOs, may interact to produce synergistic, additive or antagonistic effects. Synergistic interactions could result in increased efficacy, or reduce effective doses, therefore reducing the likelihood of adverse effects [27]. We have previously shown that pairwise combinations of polyphenols present in almonds (protocatechuic acid, naringenin and epicatechin) showed both synergistic and indifferent interactions against Salmonella enterica and Staphylococcus aureus [28].

In the present study, the interactions between pistachio hull EO and the selected antifungal compounds were antagonistic (FIC index $>4$ ) and droplet size distribution was used to investigate the mechanisms responsible for such interactions. PdI values close to 1.0 are indicative of polydispersed systems [29], whereas values close to 0.6 suggesting monomodal systems. Interestingly, amongst the antifungal used, caspofungin is the most hydrophilic one as shown by its $\log P$ value of -4.59 . This property is due to the numerous hydroxyl groups, which makes it able to donate and accept 16 and 18 hydrogen bonds respectively (Fig. 2c). This allows an excellent dispersion of the molecule in the aqueous medium leading to a 


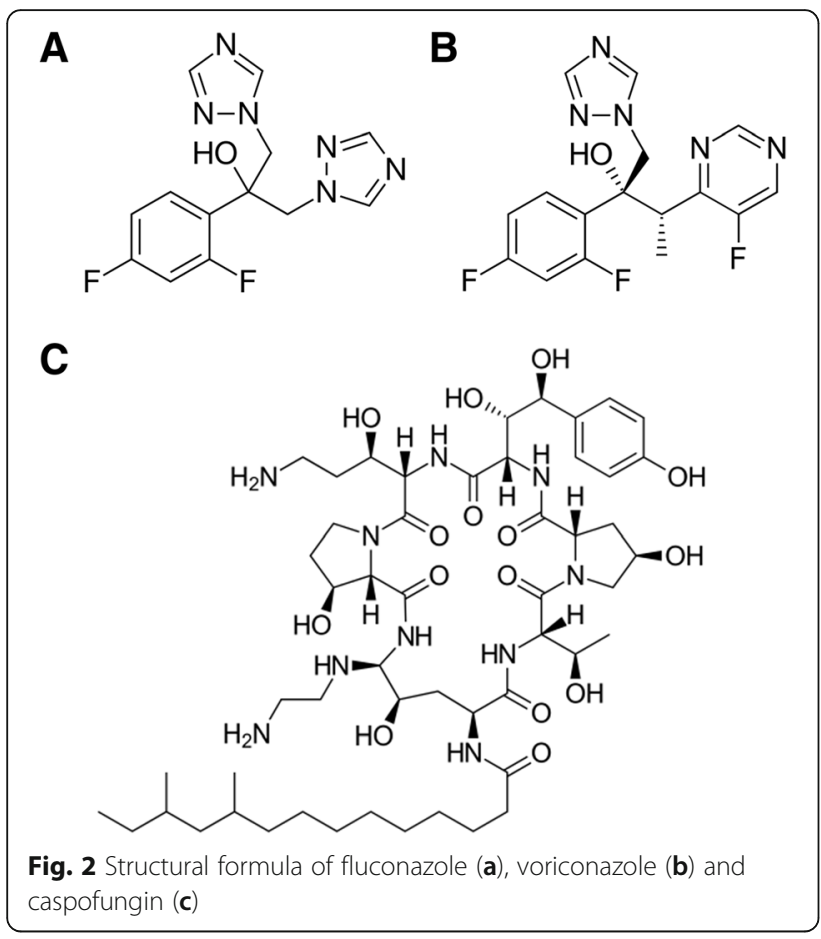

homogeneous droplet system. In fact, addition of pistachio hull EO to the system did not lead to substantial changes in the average diameter of the droplets or in the PdI of the culture medium. On the contrary, addition of the EO to the system containing either fluconazole or voriconazole (Fig. $2 \mathrm{a}$ and b) led to a system stabilization as highlighted by the decrease both of the droplet diameter average then of the PdI. These two drugs, which only differ for the presence of one more fluorine atom on position 5 of the pyrimidine ring and a 2-butanol as aliphatic chain instead of 2-propanol in the voriconazole (Fig. 2b), are less hydrophilic and possess a smaller steric encumbrance compared to caspofungin (Table 4). In fact, fluconazole and voriconazole are able to donate a single hydrogen bond and to accept 7 and 8 hydrogen bonds, respectively. Therefore, on the contrary of which happens in presence of caspofungin, fluconazole and voriconazole are not able to stabilize alone the system but at the same time, the addition of EO, which behaves like a non-ionic surfactant, stabilise the emulsions by reducing interfacial tension and promote steric repulsion between droplets [30]. The main mechanism for emulsion destabilization is actually associated with Ostwald ripening, which induces formation of larger droplets [31]. PdI values range from 1.0 to 0.688 and 0.426 for $\mathrm{EO}$ + fluconazole and $\mathrm{EO}$ + voriconazole, respectively, reflecting the homogeneity in the droplet size distribution of the new stabilized system. These events could be responsible for the difficulty of both EO and drugs to carry out their antifungal activity against Candida strains, which results in an antagonistic effect.

However, considering the interactions between EOs and antimicrobials, it is important to take into account the complex composition of EOs, which makes it rather difficult to predict mode of interaction, especially since the pharmacokinetic profiles are not elucidated. Therefore, the use of individual bioactives could be more commercially viable, with easier standardization in terms of activity, mechanisms of action, pharmacodynamics and pharmacokinetics.

\section{Conclusions}

In summary, the results of the present study showed that bioactives present in pistachio hulls EO are effective against a range of clinical strains of Candida, some of whom resistant to antifungal compounds. Further studies need to be performed to elucidate the mechanisms responsible for the activity and the interactions with antifungal compounds.

\section{Abbreviations}

EO: Essential oil; MFC: Minimum Fungicidal Concentration; MIC: Minimum Inhibitory Concentration

\section{Acknowledgements}

Not applicable.

\section{Funding}

This work has been funded by the University of Messina and Fondazione Imbesi (Messina, Italy). The funding body was not involved in the design of the study and collection, analysis, and interpretation of data, as well as and in writing the manuscript.

The Candida strains tested in this work were from a project funded by a grant from the Italian Ministry of Health to Orazio Romeo (Young Investigator Project, code: GR-2011-02347606).

\section{Availability of data and materials}

Data can be accessed at the University of Messina.

\section{Authors' contributions}

$\mathrm{CB}, \mathrm{MD}, \mathrm{GM}$ conceived the study, OR provided clinical Candida strains and performed molecular identification, $\mathrm{Pl}, \mathrm{CB}, \mathrm{MD}$ performed the antimicrobial assays, AS and DT carried out the essential oil extraction and characterization, RZ performed the light scattering experiments. All authors read and approved the final version of the manuscript.

Ethics approval and consent to participate

Not applicable.

\section{Consent for publication \\ Not applicable.}

\section{Competing interests}

The authors declared that they have no competing interests.

\section{Publisher's Note}

Springer Nature remains neutral with regard to jurisdictional claims in published maps and institutional affiliations.

\section{Author details}

'Department of Chemical, Biological, Pharmaceutical and Environmental Science, University of Messina, Via SS. Annunziata, 98168 Messina, Italy. 
${ }^{2}$ Department of Biomedical, Dental, Morphological and Functional Images Sciences, University of Messina, Via C. Valeria, 98125 Messina, Italy.

${ }^{3}$ Fondazione Imbesi, Messina, Italy. ${ }^{4}$ IRCCS Centro, Neurolesi Bonino-Pulejo, S.S. 113 Via Palermo C.da Casazza, 98123 Messina, Italy.

Received: 28 September 2018 Accepted: 26 December 2018 Published online: 07 January 2019

\section{References}

1. Yapar N. Epidemiology and risk factors for invasive candidiasis. Ther Clin Risk Manag. 2014;10:95-105.

2. Colombo AL, Júnior JNA, Guinea J. Emerging multidrug-resistant Candida species. Curr Opin Infect Dis. 2017:6:528-38.

3. Essid R, Hammami M, Gharbi D, et al. Antifungal mechanism of the combination of Cinnamomum verum and Pelargonium graveolens essential oils with fluconazole against pathogenic Candida strains. Appl Microbiol Biotechnol. 2017;101:6993-7006.

4. Swamy MK, Akhtar MS, Sinniah UR. Antimicrobial properties of plant essential oils against human pathogens and their mode of action: an updated review. Evid Based Complement Alternat Med. 2016:3012462.

5. Gucwa K, Milewski S, Dymerski T, Szweda P. Investigation of the antifungal activity and mode of action of Thymus vulgaris, Citrus limonum, Pelargonium graveolens, Cinnamomum cassia, Ocimum basilicum, and Eugenia caryophyllus essential oils. Molecules. 2018;23:E1116.

6. Dos Santos JFS, Rocha JE, Bezerra CF, et al. Chemical composition, antifungal activity and potential anti-virulence evaluation of the Eugenia uniflora essential oil against Candida spp. Food Chem. 2018;261:233-9.

7. Barreca D, Laganà G, Leuzzi U, et al. Evaluation of the nutraceutical, antioxidant and cytoprotective properties of ripe pistachio (Pistacia vera $\mathrm{L}$. variety Bronte) hulls. Food Chem. 2016:196:493-502.

8. Bellocco E, Barreca D, Laganà G, et al. Cyanidin-3-O-galactoside in ripe pistachio (Pistachia vera $L$. variety Bronte) hulls: identification and evaluation of its antioxidant and cytoprotective activities. J Func Food. 2016;27:376-85.

9. Smeriglio A, Denaro M, Barreca $D$, et al. In vitro evaluation of the antioxidant, Cytoprotective, and antimicrobial properties of essential oil from Pistacia vera L. variety Bronte Hull. Int J Mol Sci. 2017;18:E1212.

10. Romeo O, Criseo G. First molecular method for discriminating between Candida africana, Candida albicans, and Candida dubliniensis by using hwp1 gene. Diagn Microbiol Infect Dis. 2008;62:230-3.

11. Romeo O, Scordino F, Pernice I, et al. A multiplex PCR protocol for rapid identification of Candida glabrata and its phylogenetically related species Candida nivariensis and Candida bracarensis. J Microbiol Methods. 2009;79: 117-20.

12. Asadzadeh M, Ahmad S, Hagen F, et al. Simple, low-cost detection of Candida parapsilosis complex isolates and molecular fingerprinting of Candida orthopsilosis strains in Kuwait by ITS region sequencing and amplified fragment length polymorphism analysis. PLoS One. 2015;10: e0142880.

13. Müller FM, Werner KE, Kasai M, et al. Rapid extraction of genomic DNA from medically important yeasts and filamentous fungi by high-speed cell disruption. J Clin Microbiol. 1998;36:1625-9.

14. Clinical and laboratory Standards Institute, Reference method for broth dilution antifungal susceptibility testing of yeasts, approved standard M27A3, Clinical and laboratory standards institute, Wayne, Penn, USA, 3rd ed edition, 2008

15. White RL, Burgess DS, Manduru M, Bosso JA. Comparison of three different in vitro methods of detecting synergy: time-kill, checkerboard, and E test. Antimicrob Agents Chemother. 1996:40:1914-8.

16. Filocamo A, Bisignano C, D'Arrigo M, et al. Norfloxacin and ursolic acid: in vitro association and postantibiotic effect against Staphylococcus aureus. Lett Appl Microbiol. 2011;53:193-7.

17. Visalli MA, Jacobs MR, Appelbaum PC. Activities of three quinolones, alone and in combination with extended-spectrum cephalosporins or gentamicin against Stenotrophomonas maltophilia. Antimicrob Agents Chemother. 1998; 42:2002-5

18. International Standard ISO 22412:2017. Particle size analysis -- dynamic light scattering (DLS), International Organisation for Standardisation (ISO).

19. MiLogP2.2, 2018. Molinspiration property calculation service FAQ, http:// www.molinspiration.com. Accessed June 2018.

20. O'Neill J. 2016. Tackling drug-resistant infections globally: final report and recommendations. London (UK): HM Government and the Wellcome Trust.
21. Leite MC, de Brito Bezerra AP, de Sousa JP, de Oliveira Lima E. Investigating the antifungal activity and mechanism(s) of geraniol against Candida albicans strains. Med Mycol. 2015;53:275-84.

22. Ebani W, Nardoni S, Bertelloni F, et al. Activity of Salvia dolomitica and Salvia somalensis essential oils against bacteria, molds and yeasts. Molecules. 2018;23:E396.

23. Bisignano C, Filocamo A, Faulks RM, Mandalari G. In vitro antimicrobial activity of pistachio (Pistacia vera L.) polyphenols. FEMS Microbiol Lett. 2013; 341:62-7.

24. Filocamo A, Bisignano C, Mandalari G, Navarra M. In Vitro Antimicrobial Activity and Effect on Biofilm Production of a White Grape Juice (Vitis vinifera) Extract. Evid Based Complement Alternat Med. 2015;2015:856243.

25. Palá-Paúl, J, Usano-Alemany, J, Granda, E, Soria, AC: Chemical composition, antifungal and antibacterial activity of the essential oil of Chamaecyparis nootkatensis from Spain. Nat Prod Commun. 2009: 7:1007-1010.

26. Castanheira M, Deshpande LM, Davis AP, Rhomberg PR, Pfaller MA. Monitoring Antifungal Resistance in a Global Collection of Invasive Yeasts and Molds: Application of CLSI Epidemiological Cutoff Values and WholeGenome Sequencing Analysis for Detection of Azole Resistance in Candida albicans. Antimicrob Agents Chemother. 2017;61:e00906-17.

27. de Castro RD, de Souza TMPA, Bezerra LMD, Ferreira GLS, de Brito Costa EMM, Leite Cavalcanti A. Antifungal activity and mode of action of thymol and its synergism with nystatin against Candida species involved with infections in the oral cavity: an in vitro study. BMC Complement Alter Med. 2015;15:417

28. Mandalari G, Bisignano C, D'Arrigo M, et al. Antimicrobial potential of polyphenols extracted from almond skins. Lett Appl Microbiol. 2010;51:83-9.

29. Bernardi D, Pereira S, Maciel TA, et al. Formation and stability of oil-in-water nanoemulsions containing rice bran oil: in vitro and in vivo assessments. J Nanobiotechnology. 2011;9:44.

30. Wang L, Dong J, Chen J, et al. Design and optimization of a new selfnanoemulsifying drug delivery system. J Colloid Interface Sci. 2009:330:443-8.

31. Solans C, Solé I. Nano-emulsions: formation by low-energy methods. Curr Opin Colloid Interface Sci. 2012;17:246-54.

\section{Ready to submit your research? Choose BMC and benefit from:}

- fast, convenient online submission

- thorough peer review by experienced researchers in your field

- rapid publication on acceptance

- support for research data, including large and complex data types

- gold Open Access which fosters wider collaboration and increased citations

- maximum visibility for your research: over 100M website views per year

At BMC, research is always in progress.

Learn more biomedcentral.com/submissions 